\title{
Influência do dreno pleural sobre a dor, capacidade vital e teste de caminhada de seis minutos em pacientes submetidos à ressecção pulmonar*
}

\author{
Influence of pleural drainage on postoperative pain, vital capacity \\ and six-minute walk test after pulmonary resection
}

\author{
Vanessa Pereira de Lima', Daniela Bonfim ${ }^{1}$, Thais Telles Risso', Denise de Moraes Paisani ${ }^{1}$, \\ Julio Flavio Fiore Junior ${ }^{2}$, Luciana Dias Chiavegato 3 , Sonia Maria Faresin ${ }^{4}$
}

\begin{abstract}
Resumo
Objetivo: Avaliar a influência do dreno pleural sobre a distância percorrida no teste de caminhada de seis minutos, da intensidade da dor e da capacidade vital de pacientes submetidos à ressecção pulmonar. Métodos: Foram avaliados treze pacientes consecutivos, internados na Enfermaria da Cirurgia de Tórax do Hospital São Paulo, submetidos à drenagem pleural fechada (dreno tubular multiperfurado de 0,5 polegada), no período pós-operatório de ressecção pulmonar (lobectomia, segmentectomia e nodulectomia). A opção pela retirada do dreno seguiu critérios clínicos definidos por médicos da equipe cirúrgica alheios ao estudo. A determinação da capacidade vital, da intensidade da dor através da escala visual analógica de dor e da distância percorrida no teste de caminhada de seis minutos foram realizadas $30 \mathrm{~min}$ antes da retirada do dreno e $30 \mathrm{~min}$ após. A análise estatística dos dados foi realizada através do teste t pareado, com nível de significância estabelecido em 0,05. Resultados: Após a retirada do dreno, os valores obtidos na avaliação da escala visual analógica de dor foram significativamente menores $(3,46 \mathrm{~cm} v s .1,77 \mathrm{~cm} ; \mathrm{p}=0,001)$, e a distância percorrida no teste de caminhada de seis minutos foi significativamente maior $(374,34 \mathrm{~m}$ vs. 444,62 m; $\mathrm{p}=0,03)$. A capacidade vital antes e após a retirada do dreno não foi alterada de forma significativa (2,15 L vs. 2,25 L, respectivamente; $p=0,540$ ). Conclusões: Os resultados deste estudo sugerem que a presença do dreno pleural é um importante fator associado à dor pós-operatória e à limitação funcional em pacientes submetidos à ressecção pulmonar.
\end{abstract}

Descritores: Cirurgia torácica; Teste de esforço; Testes de função respiratória; Dor pós-operatória.

\begin{abstract}
Objective: To evaluate the influence of pleural drainage on the distance covered on the six-minute walk test, pain intensity and vital capacity in patients submitted to pulmonary resection. Methods: Thirteen consecutive patients from the Thoracic Surgery Infirmary of Hospital São Paulo, Brazil, submitted to closed pleural drainage (0.5-in multiperforated chest tube) in the postoperative period following pulmonary resection (lobectomy, segmentectomy and pulmonary nodule resection) were evaluated. The decision for chest tube removal followed clinical criteria defined by the surgical team, who did not participate in the study. Vital capacity, pain intensity (using a visual analog pain scale) and the distance covered on the six-minute walk test were determined $30 \mathrm{~min}$ prior to and $30 \mathrm{~min}$ after the removal of the chest tube. The statistical analysis was performed using paired t-tests, and the level of significance was set at 0.05. Results: After the removal of the chest tube, the visual analog scale pain scores were significantly lower $(3.46 \mathrm{~cm} v s .1 .77 \mathrm{~cm} ; \mathrm{p}=0.001)$ and the distance covered on the six-minute walk test was significantly higher ( $374.34 \mathrm{~m}$ vs. $444.62 \mathrm{~m} ; \mathrm{p}=0.03$ ). Vital capacity prior to and after chest tube removal was not significantly affected $(2.15 \mathrm{~L}$ and $2.25 \mathrm{~L}$, respectively; $\mathrm{p}=0.540)$. Conclusions: The results of the present study suggest that the presence of a chest tube is a factor significantly associated with postoperative pain and functional limitation in patients submitted to pulmonary resection.
\end{abstract}

Keywords: Thoracic surgery; Exercise test; Respiratory function tests; Pain, postoperative.

* Trabalho realizado na Disciplina de Pneumologia. Universidade Federal de São Paulo/Escola Paulista de Medicina - UNIFESP/EPM - São Paulo (SP) Brasil. 1. Supervisora do Curso de Especialização em Fisioterapia Respiratória. Universidade Federal de São Paulo/Escola Paulista de Medicina - UNIFESP/EPM - São Paulo (SP) Brasil.

2. Professor do Curso de Graduação em Fisioterapia. Universidade Cidade de São Paulo, São Paulo (SP) Brasil.

3. Co-coordenadora do Curso de Especialização em Fisioterapia Respiratória. Universidade Federal de São Paulo/Escola Paulista de Medicina - UNIFESP/EPM São Paulo (SP) Brasil.

4. Coordenadora do Ambulatório de Risco Cirúrgico da Disciplina de Pneumologia. Universidade Federal de São Paulo/Escola Paulista de Medicina - UNIFESP/ EPM - São Paulo (SP) Brasil.

Endereço para correspondência: Sonia Maria Faresin. Universidade Federal de São Paulo, Departamento de Medicina, Disciplina de Pneumologia, Rua Botucatu, 740, $3^{\circ}$ andar, Vila Clementino, CEP 04023-062, São Paulo, SP, Brasil.

Tel 5511 5549-1830. E-mail: faresins@pneumo.epm.br

Apoio financeiro: Nenhum.

Trabalho realizado em 17/3/2008. Aprovado, após revisão, em 9/4/2008. 


\section{Introdução}

Em procedimentos operatórios realizados no interior da cavidade torácica, o uso da drenagem pleural intercostal é freqüente. Dentre as diversas técnicas ou estratégias de drenagem que podem ser utilizadas, a mais comum consiste no uso de dois drenos torácicos; um anterior, posicionado no terço superior da cavidade torácica, e outro posterior, posicionado no terço inferior. Estudos recentes, no entanto, mostram que o uso de apenas um dreno apresenta algumas vantagens, como a redução da dor e menor custo de internação hospitalar. ${ }^{(1,2)}$

Alguns autores sugerem que a drenagem pleural intercostal, independentemente da técnica utilizada, pode ser um importante fator associado ao desenvolvimento de complicações pulmonares pós-operatórias. 0 trauma torácico decorrente da inserção do dreno pleural promoveria aumento da dor ${ }^{(3)} \mathrm{e}$ alterações adicionais da mecânica respiratória no período pós-operatório. ${ }^{(4,5)}$ Além disso, a capacidade do paciente de sair do leito precocemente após a cirurgia parece também ser prejudicada ou retardada pela presença do dreno pleural. ${ }^{(6)}$

A literatura atual sugere que a remoção precoce do dreno torácico pode ser realizada de forma segura $^{(7)}$ e gera importantes benefícios ao paciente, como a diminuição da dor torácica pós-operatória, redução na incidência de complicações pulmonares e redução do tempo de internação hospitalar. ${ }^{(8-10)} 0$ restabelecimento precoce de atividades funcionais após a remoção do dreno, como a deambulação, é apontada como um dos fatores que levariam a melhor recuperação do paciente. ${ }^{(6)}$ Existem, no entanto, poucos estudos na literatura avaliando se a remoção do dreno torácico realmente favorece a

Tabela 1 - Características dos pacientes estudados ( $\mathrm{n}=13)$.

\begin{tabular}{|c|c|}
\hline Sexo, n (\%) & \\
\hline Masculino & $9(69)$ \\
\hline Feminino & $4(31)$ \\
\hline \multicolumn{2}{|l|}{ Tipo de cirurgia, n (\%) } \\
\hline Lobectomia & $5(38)$ \\
\hline Segmentectomia & $4(31)$ \\
\hline Nodulectomia & $4(31)$ \\
\hline ldade, anos ${ }^{\mathrm{a}}$ & $59,08 \pm 11,36$ \\
\hline Tempo de drenagem torácica, dias & $3,38 \pm 0,65$ \\
\hline
\end{tabular}

realização de atividades funcionais no período pósoperatório. 0 efeito agudo da retirada do dreno sobre a dor torácica referida pelo paciente e sobre a mecânica pulmonar também foi pouco estudado. 0 presente estudo foi proposto com o objetivo de analisar a influência da remoção do dreno pleural sobre a distância percorrida no teste de caminhada de seis minutos (TC6), intensidade da dor e capacidade vital (CV) de pacientes submetidos à cirurgia de ressecção pulmonar.

\section{Métodos}

\section{Delineamento do estudo}

Estudo de coorte prospectivo realizado na Enfermaria da Cirurgia de Tórax do Hospital São Paulo, Universidade Federal de São Paulo. Foram considerados para inclusão no estudo todos os pacientes com idade $\geq 18$ anos, submetidos à cirurgia de ressecção pulmonar (nodulectomia, segmentectomia, lobectomia) e com uso de drenagem pleural fechada no período pós-operatório. Foram excluídos do estudo pacientes que apresentavam alterações ortopédicas, neurológicas ou vasculares que pudessem prejudicar a realização do TC6, assim como aqueles com incapacidade de compreender as instruções para os testes propostos.

0 cálculo da amostra foi realizado considerando a distância percorrida no TC6 como medida de desfecho principal. Para um alfa bidirecional igual a 0,05 e um poder de 80\%, considerando o desvio-padrão da distância caminhada no TC6 obtida em um estudo piloto e uma diferença de $60 \mathrm{~m}$ como clinicamente significante, inferimos que uma amostra de 13 pacientes era suficiente para responder a pergunta do estudo.

0 estudo foi aprovado pelo Comitê de Ética em Pesquisa da Universidade Federal de São Paulo/ Hospital São Paulo, e um termo de consentimento livre e esclarecido foi obtido de cada participante.

\section{Protocolo}

Todos os pacientes foram submetidos à avaliação do TC6 no período pré-operatório, conforme a rotina do serviço de cirurgia torácica da nossa instituição.

No período pós-operatório, a opção pela retirada do dreno pleural seguiu os critérios da equipe de cirurgia torácica do Hospital São Paulo, alheia 
ao estudo. A avaliação da CV, a aplicação de uma escala visual analógica para avaliar a intensidade de dor e o TC6 foram realizados $30 \mathrm{~min}$ antes da retirada do dreno e repetidos 30 min após.

A dor foi mensurada através de uma escala visual analógica. Esta escala utiliza uma linha de dez centímetros cuja extremidade esquerda indica ausência de dor e a extremidade direita indica a pior dor que o paciente já sentiu. 0 paciente foi orientado a assinalar com um traço vertical a intensidade da sua dor entre as extremidades da linha. ${ }^{(11)}$ A avaliação da dor foi realizada antes da realização do TC6 e da mensuração da CV.

A CV foi medida através de um ventilômetro (modelo 121; Ohmeda, Boulder, CO, EUA), conectado a um bocal plástico rígido, e determinada a partir de uma inspiração até a capacidade pulmonar total, seguida de uma expiração até o volume residual. Um obturador nasal foi utilizado para evitar o escape de ar pelo nariz do paciente. As medidas foram realizadas por no máximo seis vezes, até que fossem obtidos três valores com variação menor que $5 \%$, sendo considerado para a análise o maior valor obtido. Os pacientes foram avaliados na posição sentada. 0 avaliador era responsável por evitar qualquer tipo de vazamento, otimizando o ajuste do bocal nos lábios do paciente.

0 TC6 é um teste simples e reprodutível para avaliação da capacidade funcional. No presente estudo, a realização do teste seguiu os critérios da American Thoracic Society. ${ }^{(12)}$ Os pacientes foram instruídos a caminhar durante 6 min, o mais rápido possível, por um corredor de $20 \mathrm{~m}$, sem correr ou trotar. 0 fisioterapeuta encorajava o paciente, a cada minuto, usando uma ou duas frases: "você está indo bem" ou "mantenha o seu ritmo". Os pacientes foram orientados sobre a possibilidade de interromper o teste para descansar e instruídos a reiniciar a caminhada assim que se sentissem aptos, sem que houvesse interrupção da contagem do tempo.

\section{Análise estatística}

A análise estatística foi realizada com uso do programa Statistical Package for the Social Sciences para Windows, versão 13.0 (SPSS Inc., Chicago, IL, EUA). Para a análise descritiva das variáveis numéricas, foram utilizados média e desvio-padrão; para as variáveis categóricas, freqüência absoluta e porcentagem. Os coeficientes de achatamento das curvas geradas foram calculados, mostrando que os dados da CV, da escala visual analógica de dor e da distância percorrida no TC6 apresentavam distribuição normal e, portanto, deveriam ser analisados através de testes paramétricos. 0 teste $t$ pareado foi utilizado para comparar os dados obtidos antes e após a retirada do dreno pleural. 0 erro alfa foi estabelecido em 0,05.

\section{Resultados}

Foram avaliados 13 pacientes consecutivos, submetidos à ressecção pulmonar através de incisão póstero-lateral. Todos os pacientes foram submetidos à drenagem pleural fechada com um dreno tubular multiperfurado de meia polegada posicionado no quinto ou sexto espaço intercostal em região axilar média. Todos os pacientes receberam opióides através de cateter peridural até o terceiro dia do pós-operatório. Após este período, o regime de analgesia consistiu no uso de dipirona ou tramadol por via oral.

Nenhum paciente se enquadrou nos critérios de exclusão do estudo durante o período de coleta dos dados. As características dos pacientes estudados estão descritas na Tabela 1.

Os valores obtidos na escala visual analógica para avaliação da dor foram significativamente menores após a retirada do dreno $(3,46 \mathrm{~cm}$ vs. $1,77 \mathrm{~cm}$;

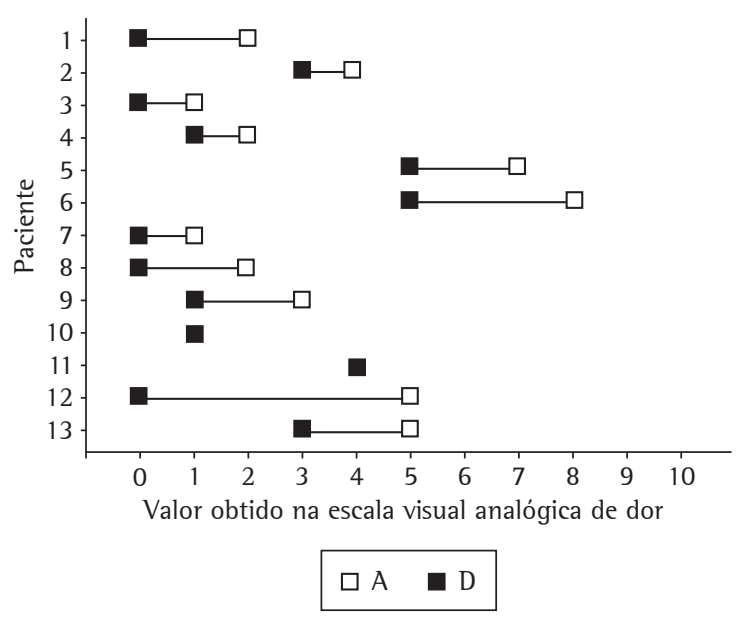

Figura 1 - Valores absolutos da escala visual analógica de dor obtidos de cada paciente antes (A) e depois (D) da remoção do dreno torácico. 


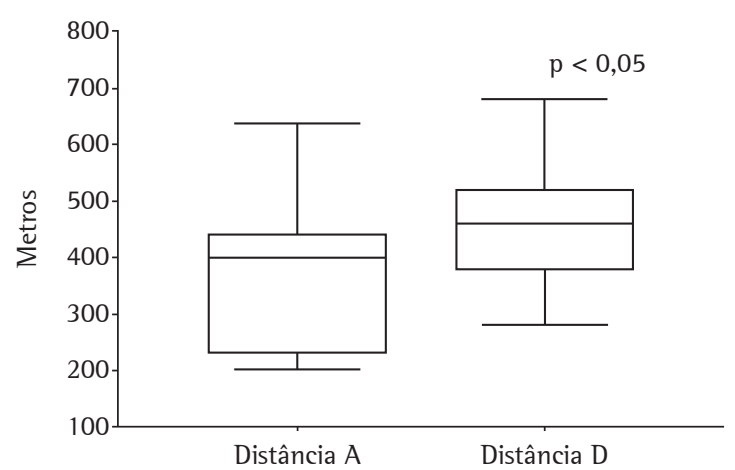

Figura 2 - Disposição gráfica do tipo box plot mostrando os dados referentes à distância percorrida no teste de caminhada de seis minutos antes (A) e depois (D) da remoção do dreno torácico.

$p=0,001)$, o que representa uma melhora de 49,7\% na dor referida pelos doentes (Figura 1).

A distância percorrida no TC6 foi significativamente maior após a retirada do dreno $(374,34 \mathrm{~m} v \mathrm{~s}$. $444,62 \mathrm{~m} ; \mathrm{p}=0,03)$. Portanto, a média da distância percorrida durante o teste após a remoção do dreno aumentou em 70,28 m (Figura 2).

Os valores obtidos para a variável CV apresentaram um discreto aumento após a retirada do dreno, porém sem significância estatística $(2,15 \mathrm{~L}$ vs. $2,25 \mathrm{~L} ; \mathrm{p}=0,54)$.

\section{Discussão}

Observamos, através deste estudo de coorte prospectivo, que a remoção do dreno pleural promove a melhora da dor torácica e favorece o desempenho no TC6 em pacientes submetidos à ressecção pulmonar. A mecânica pulmonar, avaliada através da CV, não foi influenciada pela retirada do dreno.

A redução da dor após a retirada do dreno pleural corrobora dados já observados na literatura. ${ }^{(2,3,10)}$ Alguns autores estudaram a variação da pontuação na escala visual de dor antes e após a administração de analgésicos e concluíram que reduções de 33\% nos níveis de dor representam uma diferença clinicamente significativa. ${ }^{(11)}$ No presente estudo, observamos uma redução de 49,7\% na dor após a remoção do dreno, diferença que, portanto, pode ser considerada clinicamente importante. A dor torácica é o principal motivo de queixa do paciente nos primeiros dias após a cirurgia; deste modo, além de um protocolo adequado de analgesia, qualquer outra estratégia que possa diminuir a dor torácica no período pós-operatório, como a retirada precoce do dreno torácico, deve ser aplicada sempre que possivel.

Não foi observada uma diferença significativa entre os valores de CV obtidos antes e após a retirada do dreno; porém, acreditamos que número de pacientes envolvidos no estudo não forneceu uma amostra com poder suficiente para a avaliação inferencial adequada desta variável. É possível que a tendência de aumento da CV após a retirada do dreno viesse a apresentar significância estatística com o aumento do tamanho da amostra. No entanto, alguns autores já estudaram o efeito da drenagem pleural sobre a recuperação da CV no pósoperatório de ressecção e observaram que essa não era influenciada pela remoção precoce do dreno. ${ }^{(6)}$

Para avaliar se a remoção do dreno teria alguma influência na capacidade funcional dos pacientes submetidos à ressecção pulmonar, optamos pelo uso do TC6. Trata-se de um teste simples, bem padronizado e recomendado para avaliar o efeito de intervenções terapêuticas em diversas situações clínicas. ${ }^{(12)}$ Ao contrário de outros testes de exercício, o TC6 avalia a capacidade funcional em níveis submáximos e, portanto, é considerado o teste que melhor representa a habilidade do paciente de realizar atividades de vida diária. ${ }^{(12)}$ Em nosso estudo, observamos que a distância percorrida no TC6 foi significativamente maior após a remoção do dreno torácico. Houve um incremento médio de 70,28 m em relação ao valor obtido antes da retirada do dreno, distância considerada clinicamente significante pelas diretrizes da American Thoracic Society. ${ }^{(12)} 0$ aumento na distância percorrida durante o teste pode estar relacionado com a ausência da restrição causada pelo dreno torácico durante a marcha, redução da dor torácica ou fatores psicossomáticos, tais como medo e receio de deslocar o dreno. Alguns autores sugerem que o restabelecimento precoce da deambulação após a cirurgia pode reduzir a incidência de complicações pulmonares no período pós-operatório, ${ }^{(13,14)}$ favorecer a função circulatória e diminuir a incidência de fenômenos tromboembólicos. ${ }^{(15)}$ Além disso, a deambulação poderia evitar o processo de descondicionamento físico sofrido durante a hospitalização, ${ }^{(16)}$ favorecendo o retorno precoce do paciente às suas atividades de vida diária após a alta hospitalar e a melhora de sua qualidade de vida no pós-operatório. 
Em 2001, alguns autores avaliaram o efeito da remoção precoce do dreno torácico sobre o desempenho no TC6 no pós-operatório de ressecção pulmonar. ${ }^{(6)}$ A distância percorrida no TC6, avaliado uma semana após a cirurgia, foi comparada entre um grupo de pacientes submetido à remoção precoce do dreno e outro grupo de pacientes submetido à abordagem tradicional (tempo médio de uso do dreno, 1,5 vs. 2,8 dias, respectivamente). 0 desempenho no teste foi melhor no grupo cujo dreno foi removido mais cedo. Os autores sugerem que o fato do paciente ser capaz de sair do leito e deambular precocemente é responsável pela sua melhor capacidade funcional uma semana após a cirurgia. Os resultados do presente estudo confirmam essa hipótese, mostrando que a presença do dreno torácico é um importante fator de limitação, e que a capacidade dos pacientes de realizar atividades funcionais melhora logo após a sua remoção.

A generalização dos achados do presente estudo pode ser restrita devido a algumas limitações no delineamento do protocolo de pesquisa. Como as variáveis estudadas precisavam ser avaliadas antes e após a retirada do dreno, não foi possível manter cegas as diferentes condições em que os desfechos foram avaliados, tanto aos olhos do paciente como aos do avaliador. Como não era possível randomizar a ordem da avaliação, é provável que o efeito da aprendizagem do TC6 tenha contribuído com a melhora de seu desempenho após a retirada do dreno. A literatura não aponta de forma clara qual é o real grau de influência do efeito da aprendizagem sobre este teste. ${ }^{(12)}$ Contudo, acreditamos que, no caso do nosso estudo, o fato do paciente já estar familiarizado com o TC6 desde o período pré-operatório tenha minimizado esta possível influência.

Os resultados do presente estudo sugerem que a presença do dreno pleural é um importante fator associado à presença de dor e à limitação funcional em pacientes submetidos à ressecção pulmonar. Mais estudos são necessários para avaliar se o restabelecimento precoce da deambulação, favorecido pela retirada do dreno, está associado à redução da incidência de complicações pulmonares após a cirurgia, assim como se isso favorece o retorno dos pacientes às suas atividades de vida diária e influencia de forma positiva a qualidade de vida após a alta hospitalar.

\section{Referências}

1. Alex J, Ansari J, Bahalkar P, Agarwala S, Rehman MU, Saleh A, et al. Comparison of the immediate postoperative outcome of using the conventional two drains versus a single drain after lobectomy. Ann Thorac Surg. 2003;76(4):1046-9.

2. Gómez-Caro A, Roca MJ, Torres J, Cascales P, Terol E, Castañer J, et al. Successful use of a single chest drain postlobectomy instead of two classical drains: a randomized study. Eur J Cardiothorac Surg. 2006;29(4):562-6.

3. Jakob H, Kamler M, Hagl S. Doubly angled pleural drain circumventing the transcostal route relieves pain after cardiac surgery. Thorac Cardiovasc Surg. 1997;45(5):263-4.

4. Ali J, Weisel R, Layung A, Kripke B, Hechtman H. Consequences of postoperative alterations in respiratory mechanics. Am J Surg. 1974;128 (3):376-82.

5. Craig DB. Postoperative recovery of pulmonary function. Anesth Analg. 1981;60(1):46-52.

6. Nomori H, Horio $H$, Suemasu K. Early removal of chest drainage tubes and oxygen support after a lobectomy for lung cancer facilitates earlier recovery of the 6-minute walking distance. Surg Today. 2001;31(5):395-9.

7. Younes RN, Gross JL, Aguiar S, Haddad FJ, Deheinzelin D. When to remove a chest tube? A randomized study with subsequent prospective consecutive validation. J Am Coll Surg. 2002;195(5):658-62.

8. Russo L, Wiechmann RJ, Magovern JA, Szydlowski GW, Mack MJ, Naunheim KS, et al. Early chest tube removal after video-assisted thoracoscopic wedge resection of the lung. Ann Thorac Surg. 1998;66(5):1751-4.

9. Sienel W, Mueller J, Eggeling S, Thetter O, Passlick B. Early chest tube removal after video-assisted thoracoscopic surgery. Results of a prospective randomized study [Article in German]. Chirurg. 2005;76(12):1155-60.

10. Mueller XM, Tinguely F, Tevaearai HT, Ravussin P, Stumpe F, von Segesser LK. Impact of duration of chest tube drainage on pain after cardiac surgery. Eur J Cardiothorac Surg. 2000;18(5):570-4.

11. Farrar JT, Portenoy RK, Berlin JA, Kinman JL, Strom BL. Defining the clinically important difference in pain outcome measures. Pain. 2000;88(3):287-94.

12. ATS Committee on Proficiency Standards for Clinical Pulmonary Function Laboratories. ATS statement: guidelines for the six-minute walk test. Am J Respir Crit Care Med. 2002;166(1):111-7.

13. Brieger GH. Early ambulation. A study in the history of surgery. Ann Surg. 1983;197(4):443-9.

14. Mackay MR, Ellis E, Johnston C. Randomised clinical trial of physiotherapy after open abdominal surgery in high risk patients. Aust J Physiother. 2005;51(3):151-9.

15. Dean E, Ross J. Discordance between cardiopulmonary physiology and physical therapy. Toward a rational basis for practice. Chest. 1992;101(6):1694-8.

16. Suesada MM, Martins MA, Carvalho CR. Effect of short-term hospitalization on functional capacity in patients not restricted to bed. Am J Phys Med Rehabil. 2007;86(6):455-62. 